\title{
Tendencias de la multialfabetización en los albores del siglo XXI: alfabetización mediática e informacional (AMI) como propuesta integradora
}

Maylin Frías-Guzmán

Master en Bibliotecología y Ciencia de la Información, Universidad de La Habana. Diploma de Estudios Avanzados, Universidad de Granada. Doctorando en Información y Documentación, Universidad de Granada. Profesora Auxiliar, Departamento de Ciencias de la Información Cuba

http://dx.doi.org/10.1590/1981-5344/2393

La evolución de alfabetismos se produjo como resultado de la transición de una cultura oral a una cultura letrada. El presente estudio examina las principales tendencias de la multialfabetización a principios del siglo XXI. Se combina el método de análisis documental y el método bibliométrico en el análisis de la evolución de los alfabetismos, a través de documentos que establecen pautas en su fundamentación y el comportamiento de la producción científica por año en la primera década del siglo XXI. Se revela el lugar destacado que ocupan la Alfabetización Mediática y la Alfabetización Informacional con respecto al resto de las expresiones que surgen. La Alfabetización Multimedia es un alfabetismo poco abordado en la literatura como proceso de formación de competencias vinculadas a la recepción y producción de mensajes a partir de la convergencia de lenguajes y medios. La noción de Alfabetización Mediática e Informacional (AMI) constituye la principal tendencia que integra los diversos alfabetismos que promueven la participación activa en la construcción y transformación del individuo y la sociedad.

palabras clave: Multialfabetización; Alfabetización Multimedia; Alfabetización Mediática; Alfabetización Informacional; Alfabetización Medíatica e Informacional (AMI). 


\section{Multiliteracy trends in the twenty-first century: Media and Information Literacy (AMI) as integrative proposal}

The evolution of the concept of literacy was the result of the transition from an oral culture to a written one. This study examined the major trends multiliteracy early twenty-first century. Documentary analysis and bibliometric method were combined to analyze the evolution of literacy through documents that establish its foundation guidelines and the scientific production behavior per year in the first decade of the XXI century. Media Literacy and Information literacy have an important place above the rest of expression. The multimedia literacy is little addressed in the literature, as a process of training competences on reception and production of messages, from the convergence of languages and media. The notion of Media and Information Literacy (AMI) is the main trend that integrates the various literacies that promote active participation in the construction and transformation of the individual and society.

Keywords: Multiliteracies; Multimedia Literacy; Media Literacy; Information Literacy; Media and Information Literacy (MIL).

\section{Tendências da Multi-alfabetização no século XXI: Alfabetização Midiática e Informacional (AMI) como proposta integradora}

A evolução das literacias ocorre como resultado da transição de uma cultura oral para a cultura letrada. O presente estudo analisa as principais tendências da Multialfabetização início do século XXI. O método de análise de documentos e método bibliométrico na análise da evolução das literacias combinados, através de documentos que estabelecem diretrizes em seu raciocínio e do comportamento da produção científica por ano na primeira década do século. Seu principal foco Alfabetização Midiática e Alfabetização Informacional do resto das expressões que surgem lugar é revelado. 
Alfabetização Multimidia é um pouco abordado na literatura como um processo de formação de competências relacionadas com a recepção e produção de mensagens a partir da convergência de linguagens $e$ meios de comunicação. A noção de Alfabetização Midiática e Informacional (AMI) é a principal tendência que integra os vários letramentos que promovem a participação ativa na construção e transformação do indivíduo e da sociedade.

Palavras-chave: Multi-alfabetização; Alfabetização Midiática; Alfabetização Informacional; Alfabetização Multimidia; Alfabetização Midiática e Informacional.

Recebido em 10.04.2015 Aceito em 17.06.2015

\section{Introducción}

La alfabetización adquiere una connotación diferente en el marco del siglo XXI. Codificar y descodificar cualquier texto escrito, resultó insuficiente a finales del siglo $X X$, en el empeño de lograr una acción transformadora del individuo. Su concepción evolucionó desde la capacidad de leer y escribir hasta la adquisición de destrezas, habilidades, conocimiento y actitudes producto de un entorno informativo, tecnológico y mediático complejo (BAWDEN, 2002). Abogó por el aprendizaje en el seno familiar, en la calle, el vecindario, el trabajo, el mercado, y el ocio, teniendo en cuenta la dinámica del presente, el pluralismo, la comprensión mutua y el respeto a las fronteras culturales, estatales y nacionales.

En este marco, la Organización de las Naciones Unidas para la Educación, la Ciencia y la Cultura (UNESCO), desde su Sector de Comunicación e Información, en cooperación con el Sector de Educación y la Oficina Internacional de Educación, comenzó a promover a principios del siglo XXI, la concepción de Alfabetización Mediática e Informacional $(\mathrm{AMI})^{1}$. Esta propuesta contribuye a la integración, combinación y unificación, bajo un denominador común, los diversos alfabetismos que existen en la literatura.

¿Cómo han evolucionado los alfabetismos en las dos primeras décadas del siglo XXI hasta una propuesta integradora de Alfabetización Mediática e Informacional (AMI)? ¿Qué relaciones se establecen entre los diferentes alfabetismos? ¿Qué particularidades presenta la concepción de Alfabetización Mediática e Informacional?, constituyen interrogantes a revelar en el presente estudio que tiene como objetivo: examinar las principales tendencias de la multialfabetización a principios del siglo XXI.

\footnotetext{
${ }^{1}$ En inglés Media and Information Literacy (MIL).
} 


\section{Métodos}

El estudio clasifica dentro del tipo de investigación documental atendiendo al apoyo cardinal en fuentes de información documentales. Su desarrollo contempló la combinación del método de análisis documental o método bibliográfico y el método bibliométrico.

Se consultaron documentos a nivel internacional que establecen pautas en la evolución de la tipología de alfabetismos. Se insistió en los de la autoría de la UNESCO, entidad reconocida como promotora de su desarrollo.

Desde la perspectiva bibliométrica, se analizó la cantidad de publicaciones por año en el espacio temporal comprendido entre el año 2000 al 2010, en la Base de Datos "Science Citation Index", como fuente de información primaria. En el examen de la evolución de los alfabetismos los términos seleccionados se basaron en la clasificación de Tyner (1998). Se efectuaron búsquedas de cada uno de los términos de manera independiente según las ecuaciones siguientes:

a) TS= (INFORMATION LITERACY);

b) TS = (MEDIA LITERACY);

c) $T S=($ TECHNOLOGY LITERACY $)$;

d) $\mathrm{TS}=($ NETWORK LITERACY);

e)TS= (VISUAL LITERACY).

Se elaboró una nube de etiquetas con el Tagul, herramienta 2.0, que permitió la representación del contenido de las declaraciones internacionales vinculadas al desarrollo y promoción de la AMI.

\section{Resultados y discusión}

\subsection{La multialfabetización}

Las concepciones y definiciones de alfabetización son productos culturales de la postmodernidad, entendida esta última, como forma de ver el mundo. No comparten la visión del dominio exclusivo de competencias instrumentales. Insisten en la reproducción crítica de lo que se enseña, en la capacidad de dar sentido al mundo y desarrollar perspectivas propias.

En el marco de la UNESCO, se manifestó una modificación paulatina de su concepto, desde mediados del siglo XX hasta la actualidad. Las principales tradiciones, críticas y enfoques de la alfabetización se resumen en cuatro concepciones diferentes (UNESCO, 2006). Estas nociones no son excluyentes unas de otras, sino que se interrelacionan y yuxtaponen:

1 Alfabetización como conjunto autónomo de competencias;

2 Alfabetización tal como se aplica, se practica y se sitúa;

3 Alfabetización como proceso de aprendizaje; 


\section{Alfabetización como texto.}

La evolución conceptual de la alfabetización propició la proliferación de diversas locuciones. La alusión a alfabetizaciones múltiples (multiple literacies) representa un cambio del paradigma para la noción tradicional de alfabetización. Refiere a las diversas prácticas y sistemas semióticos para construir, adquirir, comunicar y demandar conocimiento, así como, para crear, analizar y transformar las relaciones entre las personas y entre estas y las instituciones (LONSDALE; MCCURRY, 2004; MCPHERSON, 2004; PAUL, 2006).

La pluralidad de los alfabetismos comprende "[...] desde la oralidad hasta la alfabetización funcional, desde las matemáticas básicas hasta el uso de ordenadores, desde la lectura hasta la Alfin" (BYRNE, 2005, p. 9). Los alfabetismos visual, audiovisual, digital, computacional, multimedia, de red, tecnológico, cultural, bibliotecario e informacional son neologismos atribuidos a las nuevas dimensiones, que adquirió la alfabetización, relacionadas con la evolución de los soportes de información y la tecnología (PONJUAN, 2002; DUDZIAK, 2003). La "alfabetización en publicidad" (PEDROSO, 2003) y "alfabetización tecnológica multimodal e intercultural" (BAUTISTA, 2007) son muestra de léxicos que se promueven eventualmente asociados a actividades y contextos específicos. "Webliteracy" (Mackey e Ho, 2005) y "cyberliteracy" (MACKEY; JACOBSON, 2011) corresponden con el uso de los ambientes Web en Internet.

Sobre la aparición cronológica de los alfabetismos, Bawden (2002, p. 363) apunta:

Alfabetización informática y alfabetización bibliotecaria han mantenido una presencia constante en la literatura, la primera con un mayor volumen que la segunda. $\mathrm{AI}^{2}$ mantuvo un volumen bajo en los 80 , extendiéndose considerablemente su uso en los 90. La baja presencia de la alfabetización en medios de comunicación se ha tornado considerablemente alta a finales de los 90, mientras que los conceptos de alfabetización en redes y alfabetización digital acaban de surgir en esta época.

Una visión de la evolución paulatina de algunos alfabetismos en la primera década del siglo XXI resulta del análisis de su producción científica. El gráfico 1 ilustra el comportamiento en el Social Science Citation Index.

Gráfico 1 - Producción científica sobre alfabetismos en el Social Science Citation Index (2000-2010)

\footnotetext{
${ }^{2}$ AI es utilizado por el autor para denominar la Alfabetización Informacional. "ALFIN es el acrónimo empleado internacionalmente para denominar este alfabetismo.
} 


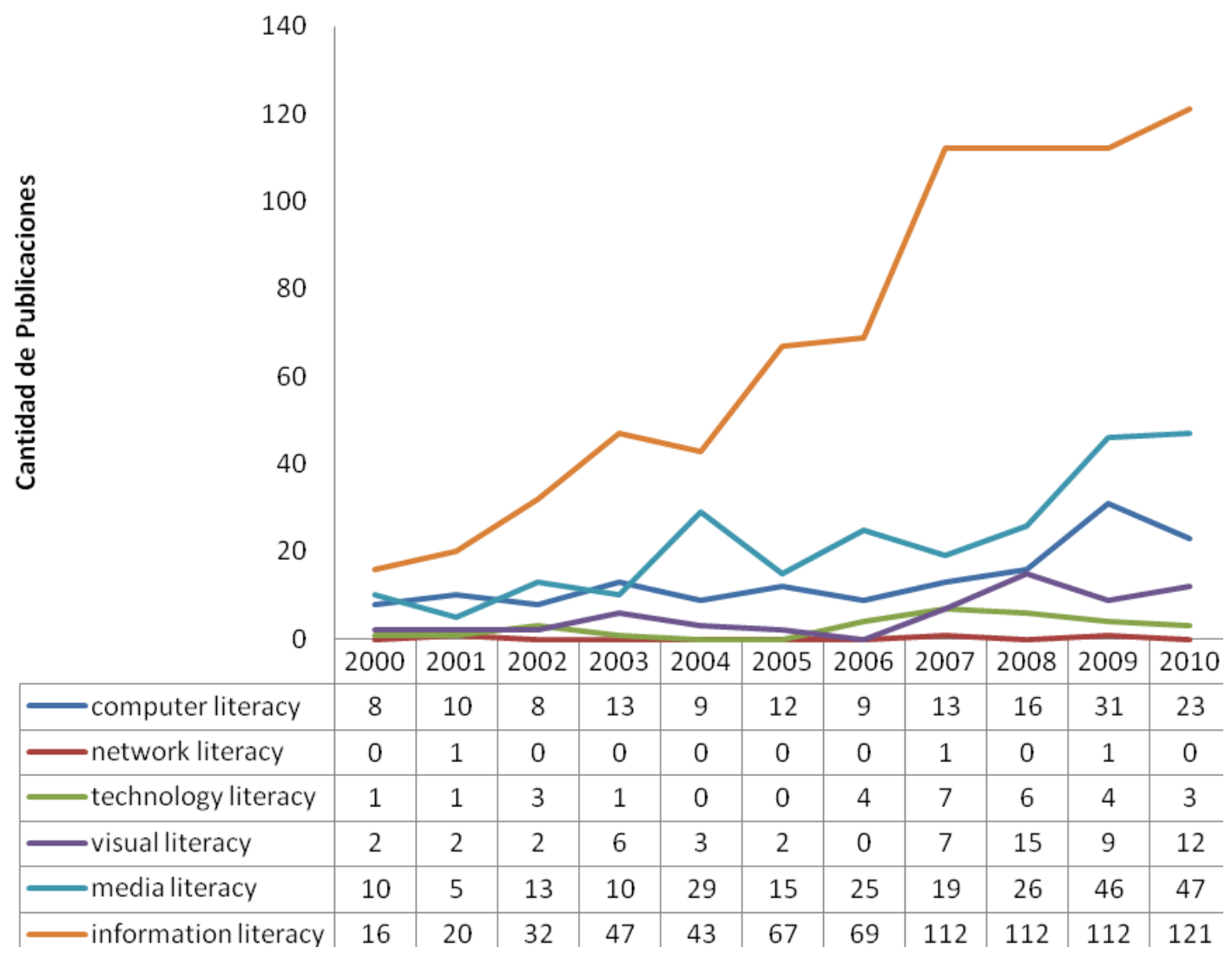

Fuente: Elaboración propia.

Las alfabetizaciones Tecnológica, de Redes e Informática muestran una escasa representación producto de su relación e inclusión como componente transversal de los alfabetismos. Para varios autores constituyen procesos más complejos que la mera capacitación en el manejo de hardware o software y enfatizan en la alfabetización ante los nuevos códigos y formas comunicativas de la cultura digital (AREA; PESSOA, 2012). La Alfabetización Informática tiene un tenue incremento con respecto a las dos primeras debido a una mayor aceptación de la expresión, en correspondencia con la expansión y aplicación de los sistemas computacionales en el procesamiento, almacenamiento y transmisión de datos e información.

El abandono de la Alfabetización Visual se justifica por su integración en la Alfabetización Mediática (MESSARIS, 1998; DONDIS, 2003; NATHARIUS, 2004; ALBERICH et al., 2005; APARICI; GARCÍA, 2008). Su ligero aumento a finales de la década se vincula al auge de esta última, su aplicación en el ámbito de las Ciencias Médicas, así como, los trabajos encaminados a la estandarización de las competencias visuales por la Asociación de Bibliotecas Universitarias y de Investigación (Association of College and Research Libraries, ACRL) (HATTWIG et al., 2013).

El impulso que presenta la Alfabetización Mediática resulta de la promoción de la educación en medios en diversos colectivos sociales y su concepción como eje clave en las democracias modernas y el "mundo de las pantallas" (KELLNER; SHARE, 2005; TYNER, 2008; AGUADED, 2009; 
HOBBS; JENSEN，2009; CABERO; GUERRA， 2011; AGUADED， 2012; 2013). Desde el siglo anterior constituyó objetivo fundamental de la Declaración de Grünwald (1982) y se reitera en el siglo XXI en la Declaración de Alejandría (2005).

La Alfabetización Informacional resalta por su primacía. El hecho se asocia al auge de la denominada Sociedad de la Información y a la concepción integradora y genérica que se le atribuye a la expresión. La Declaración de Praga (THE PRAGUE..., 2003), la Declaración de Alejandría (2005) y la Declaración de Lyon (2014) exhortaron su importancia para lograr un desarrollo humano sustentable y construir participativamente una sociedad inclusiva en el siglo XXI.

El estudio de Lee y So (2014, p. 142-143) coincide en que la Alfabetización Mediática y la Alfabetización Informacional:

[...] han experimentado un rápido desarrollo en años recientes. Esta aceleración es más evidente en los años 2000, especialmente de 2005 en adelante. En las últimas dos décadas, ambos campos se han erigido como áreas de investigación jóvenes y de potencial crecimiento dentro del ámbito de la alfabetización.

Las aproximaciones teóricos- metodológicas entre la Alfabetización Mediática e Informacional que comenzaron a aparecer en la literatura en la primera década, inciden en el comportamiento del grafo. La implicación de la UNESCO y la Federación Internacional de Asociaciones Bibliotecarias (International Federation Library Association, IFLA), en el fomento e integración de la Alfabetización Mediática y la Alfabetización Informacional, incide en el aumento de publicaciones que colocan a ambas por encima de las restantes. Las declaraciones de Fez (FEZ...2011), Moscú (DECLARACIÓN..., 2012), Paris (PARIS..., 2014) y las Recomendaciones IFLA (2011) contribuyen a la prolongación prospectiva de esta tendencia en la literatura durante la segunda década del siglo XXI, mediante la promoción, incremento y entendimiento de la conexión entre ambas.

Los análisis que han pretendido sintetizar, agrupar o integrar bajo un denominador común la multiplicidad de expresiones existentes, tienen sus primeros intentos en el campo de la pedagogía, liderado por el New London Group en 1996. El multialfabetismo (multiliteracies) fue la expresión que incluye prácticas textuales, visuales, auditivas y gestuales vinculadas a múltiples espacios y contextos culturales (CAZDEN et al., 1996).

La clasificación de Tyner diferencia las competencias relativas al manejo de las herramientas para acceder a la información de las relacionadas con el análisis e interpretación del contenido. La distribución de las seis alfabetizaciones se presenta de la siguiente forma:

a)Alfabetizaciones instrumentales: 
Alfabetización informática (Computer Literacy),

Alfabetización en redes (Network Literacy),

Alfabetización Tecnológica (Technology Literacy);

b)Alfabetizaciones representacionales:

Alfabetización Informacional (Information Literacy),

Alfabetización Visual (Visual Literacy),

Alfabetización para los medios (Media Literacy).

El estudio de Bawden partió de la ausencia de trabajos encauzados a definir exhaustivamente cada uno de los vocablos surgidos. Identificó a la Alfabetización Informacional y la Alfabetización Digital como conceptos abarcadores y estrechamente vinculados. Ambas propuestas descansan sobre las base de otras alfabetizaciones comúnmente difundidas que agrupó en dos categorías. Las alfabetizaciones basadas en destrezas incluyen las relacionadas con el desarrollo de habilidades técnicas. Las alfabetizaciones digitales, las que hacen énfasis en la comprensión crítica del contenido y el contexto:

Pasadas sintetizó las propuestas relativas sobre el término alfabetización teniendo en cuenta, los segmentos estructurales del modelo de comunicación (mensajes, modos, medios y soportes). Sus tres categorías abarcan la noción de preparación cultural general e integral; de desarrollo de competencias asociadas al continente y el contenido y por último el dominio de modos, códigos y medios de comunicación, desde los tradicionales hasta los que aparecen en el escenario actual:

a)alfabetismos como conjuntos de conocimientos fundamentales sobre un tema o cuestión relevante;

b)alfabetismos como metáforas de habilidades y competencias;

c)alfabetismos en sentido estricto (PASADAS, 2008).

Desde una perspectiva integradora, se intenta combinar y unificar la variedad de alfabetismos, como punto divergente de las nociones tradicionales de segmentación. Las dimensiones instrumental y básica, emocional, ética y social se encierran en la proposición de alfabetización múltiple o multimodal (GUTIÉRREZ, 2000a; BAWDEN, 2002; CORDES, 2009; PINTO et al., 2009). La similitud entre las prácticas actuales y las antiguas de lectura y escritura, con la atenuante que introduce la evolución tecnológica en los canales y plataformas de comunicación, derivan en la propuesta del término "transliteracy" (THOMAS et al., 2007; ANDRETTA, 2009). La propuesta nórdica de "'electracy" resulta de la intersección de la alfabetización crítica con la Alfabetización en medios, la Alfabetización Multimedia, la Alfabetización 
Informática, la Alfabetización Informacional y la Alfabetización Visual (ERSTAD, 2003).

Una de las tendencias más aceptadas y extendidas en los especialistas del tema es la de considerar a la Alfabetización Informacional como metalfabetización, competencia genérica o concepto paraguas bajo el cual se recogen las otras alfabetizaciones (KULTHAU, 1993; BAWDEN, 2002; PONJUAN, 2002; SANTOS, ANDRADE et al., 2008; PINTO et al., 2009; MACKEY; JACOBSON, 2011; URIBE, 2012). Se fundamentan a partir de la naturaleza de la información.

La UNESCO presentó de manera holística una combinación de Alfabetización Mediática y Alfabetización Informacional:

[...] bajo el paraguas del término: Alfabetización Mediática e Informacional. Parte desde dónde las terminologías tienen un significado individual, [...] hacia una noción unificada que engloba los elementos tanto de Alfabetización Mediática como de Alfabetización Informacional y da a conocer las metas y objetivos de AMI" (Alfabetización Mediática e Informacional) (WILSON et. al, 2011).

En inglés, su similar corresponde con las siglas MIL (Media and Information Literacy) (Figura 1).

Figura 1 - Conceptos que componen la AMI

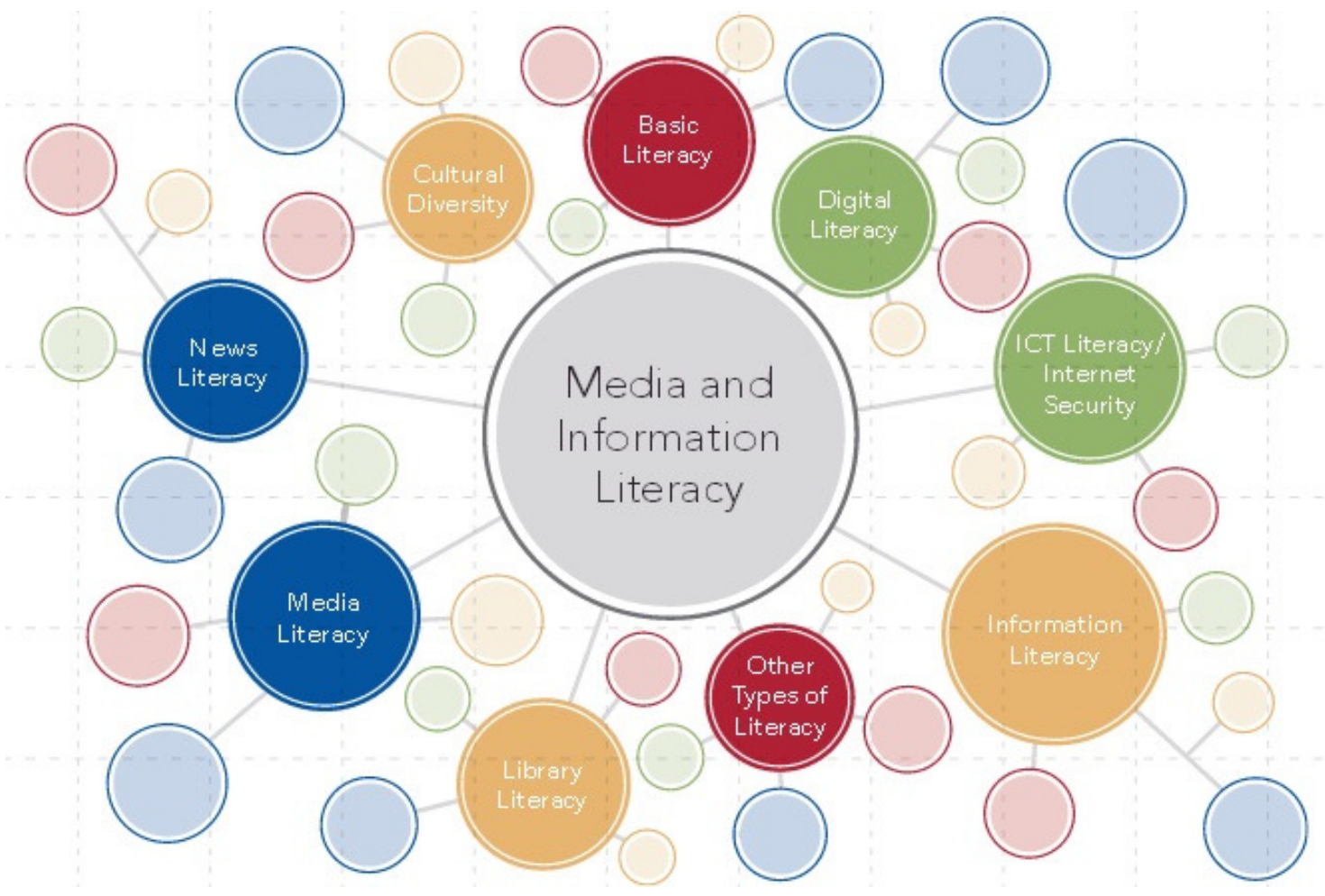

Fuente: UNESCO (2013). 
La propuesta incluye una muestra de alfabetismos que enfatiza en la importancia de la información, los medios de comunicación y las instituciones o proveedores de información, para el aprendizaje, el trabajo y la vida cotidiana.

En las dos primeras décadas del siglo XXI, el multialfabetismo manifiesta una propensión hacia la combinación, unificación y regulación de propuestas. La Alfabetización Mediática y la Alfabetización Informacional sobresalen a partir de una concepción integradora que incluye varios alfabetismos como nociones ý requisitos previos por la construcción de una sociedad del conocimiento inclusiva, abierta, participativa y pluralista.

\subsection{La Alfabetización Multimedia un alfabetismo olvidado}

La expresión Alfabetización Multimedia (Multimedia Literacy) comenzó a utilizarse alrededor de la década del 90. Las posibilidades que el entorno digital le ofreció a la fusión de diferentes formas de expresión concurrió en la presentación de "...nuevos contenidos y una manera diferente de abordar los antiguos contenidos..." (NEGROPONTE, 1995).

Las referencias al tema se encuentran en los estudios de Gutiérrez (1998a; 2000a; 2000b; 2000c; 2003a; 2003b), Hofstetter (2001), Lanham (1995), Badwen (2002), Gutiérrez y Hottmann (2002), Camargo ( s.f.), Daley (2003), Ferreiro (2005), Genzuk (2005), Aparici (2005), Lemke (1998b; 2005), Zain y Yew (2009), Hobbs (2006), Hartley (2007), Fýrat y Vicdan (2008), Hartley et al. (2008) y Pinto y Sales et al. (2009). Su proliferación ha estado limitada a partir de las relaciones de integración, yuxtaposición y subordinación con varios alfabetismos.

En ocasiones, la Alfabetización Multimedia y la Alfabetización Digital han sido considerada sinónimo o subordinación una de otra (GUTIÉRREZ, 2000a; BAWDEN, 2002; FERREIRO, 2005; HARTLEY et al., 2008; PINTO, SALES et al., 2009). Lanham (citado en Badwen, 2002), es partidario de la analogía entre ambos términos cuando se refiriere a la capacidad para leer y entender textos de hipertexto y multimedia. Sin embargo, para Bawden (2002), la Alfabetización Multimedia junto con la Alfabetización en redes, en Internet, la hiperalfabetización son consideradas manifestaciones de la Alfabetización Digital.

Gutiérrez (2000a), señala el uso del término Alfabetización Multimedia, Multimodal o Múltiple estableciendo una analogía con la Alfabetización Digital, al declarar la creación multimedia como principio básico. Las correlaciones con la Alfabetización Verbal, la Alfabetización Funcional y la Alfabetización Audiovisual son subrayadas por este autor. Su propuesta sintetiza en la Alfabetización Multimedia los objetivos y contenidos mínimos de los principios de la Alfabetización Informática, la Alfabetización en Redes, la Alfabetización Tecnológica, la Alfabetización Informacional, la Alfabetización Visual y la Alfabetización Mediática.

Las posiciones de Lanham (1995), Gutiérrez (2000a) y Daley (2003) ofrecen puntos coincidentes con la propuesta conocida como 
hiperalfabetización. La interactividad que se introduce en los multimedia repercute en una mirada particular al fenómeno.

El retorno de la Alfabetización Multimedia a la noción lecto- escritora de la Alfabetización es puntualizado por Badwen (2002). Esta visión está latente en los trabajos de Lanham (1995), Gutiérrez (2000a), Daley (2003), Genzuk (2005) y Hartley (2007). La Alfabetización Multimedia y la Alfabetización Mediática suelen ser igualadas en ocasiones (ALBERICH PASCUAL et al., 2005; HOBBS, 2006; HARTLEY, 2007) cuando se declaran a los medios de comunicación, tanto analógicos como digitales, como objetos de aprendizaje. El auge y evolución de la Alfabetización Mediática ha incidido en la integración de la Alfabetización Multimedia a su concepción y en el desuso de esta última. La atención de la Alfabetización Mediática a los medios de comunicación contemporáneos (GUTIÉRREZ; TYNER, 2012a; GUTIÉRREZ; TYNER, 2012b) desvirtúa los límites entre ambas. Una y otra contemplan el análisis crítico y creación de información en diferentes medios y formatos, lo que incluye la interpretación y uso de códigos y lenguajes, que constituye la esencia de la Alfabetización Multimedia.

La atención a los sistemas simbólicos y medios, como formas de expresión y soportes, que adquiere la información en la sociedad actual, establece la relación entre la Alfabetización Multimedia y la Alfabetización Informacional. Aunque algunos autores consideran que la Alfabetización informacional se centra en la información en general y no puntualiza en sistemas simbólicos específicos (LAU, 2013), coinciden en competencias para la evaluación y la comunicación de mensajes construidos por diferentes lenguajes y medios. La Alfabetización Multimedia profundiza más en el significado y los aspectos semánticos teniendo en cuenta los preceptos que la Alfabetización Informacional emplea en el análisis de información. Para el acceso y la representación de mensajes la Alfabetización Multimedia se auxilia de los principios instituidos por la Alfabetización Informacional.

La Alfabetización Multimedia se integra a esta concepción en la medida que incluye "[...] todos los tipos de medios (orales, impresos, analógicos y digitales) y a todas las formas de formatos y soportes" a los que se dirige. Considera el análisis, comparación y validación de "...información, mensajes, creencias y valores transmitidos a través de los medios y de toda clase de productores de contenidos [...] en base a criterios genéricos, personales y basados en sus contextos.". Incluye competencias tecnológicas y "...habilidades del aprendizaje, el pensamiento crítico y la interpretación a través de los límites profesionales, educativos y sociales." (DECLARACIÓN..., 2012).

La Alfabetización Multimedia se vincula estrechamente a la Alfabetización Digital e incluye elementos de la Alfabetización Visual y Audiovisual. Constituye una expresión de la evolución del concepto de alfabetización y el auge de alfabetismos vinculados al desarrollo de competencias para la comprensión y producción de lenguajes y medios en el contexto digital. Se complementa a partir de los principios que 
establecen la Alfabetización Mediática y la Alfabetización Informacional. Se integra a la noción de Alfabetización Mediática e Informacional (AMI) a partir de la atención de los diferentes tipos de medios, formatos y soportes, así como, la formación de competencias de pensamiento comprensivo, crítico y creativo, respetando el alcance y esencia de cada alfabetismo.

\subsection{La Alfabetización Mediática e Informacional una propuesta integradora}

La Alfabetización Mediática e Informacional se fundamenta a partir del derecho de todo ser humano a la libertad de opinión y de expresión, así como, el fomento del diálogo social entre los pueblos de todo el mundo. Sienta sus bases y objetivos en correspondencia el Desarrollo del Milenio de las Naciones Unidas (UN), la Declaración de Derechos Humanos de la Naciones Unidas y la Cumbre Mundial de la Sociedad de la Información (DECLARACIÓN..., 2012).

Su principal antecedente histórico se remonta al Simposio Internacional de UNESCO sobre la Enseñanza de los Medios, celebrado en Grünwald, Alemania en 1982. Su evolución se enmarca en el espacio temporal de las dos primeras décadas del siglo XXI a partir de la reunión de expertos de todas las regiones y diversos dominios en los que figuran los medios de comunicación, la información, la educación, el desarrollo curricular, la psicometría y las estadísticas. En la primera década se sientan las bases y la segunda marcó el inicio de un proceso dirigido a la revisión y validación de las bases conceptuales, el conjunto genérico de los medios de comunicación y las competencias necesarias.

Su reconocimiento internacional se evidencia a través de las siguientes declaraciones:

a)La Declaración Grünwald sobre la educación relativa a los medios de comunicación (1982);

b)Declaración de Praga "Hacia una sociedad alfabetizada en información" (2003);

c)Declaración de Alejandría: "Faros de la Sociedad de la Información" (2005);

d)Declaración de Fez "Alfabetización Mediática e Informacional " (2011);

e)Declaración de Moscú sobre Alfabetización Mediática e Informacional y (2012);

f)Declaración de Paris en Alfabetización Mediática e Informacional en la Era Digital (2014).

La figura 2 representa el contenido esencial de dichas declaraciones, en las que la AMI en su siglas en inglés (MIL), ocupan un lugar destacado. El uso de los medios y la información constituyen el centro del aprendizaje 
a partir de la formación de competencias o habilidades. Enfatizan en los principios básicos que fundamentan la construcción de una Sociedad de la Información que contribuirán al desarrollo social, cultural y económico de las naciones, comunidades, instituciones y personas para el siglo XXI.

Las declaraciones consideran la AMI esencial en los disímiles contextos, a partir de los niveles heterogéneos de desarrollo tecnológico. La caracterizan en función de ampliar el conocimiento de las propias necesidades vinculadas a la identificación, localización, evaluación, organización, creación, utilización de medios e información.

Figura 2 - Mapa de palabras de las declaraciones relativas a la AMI

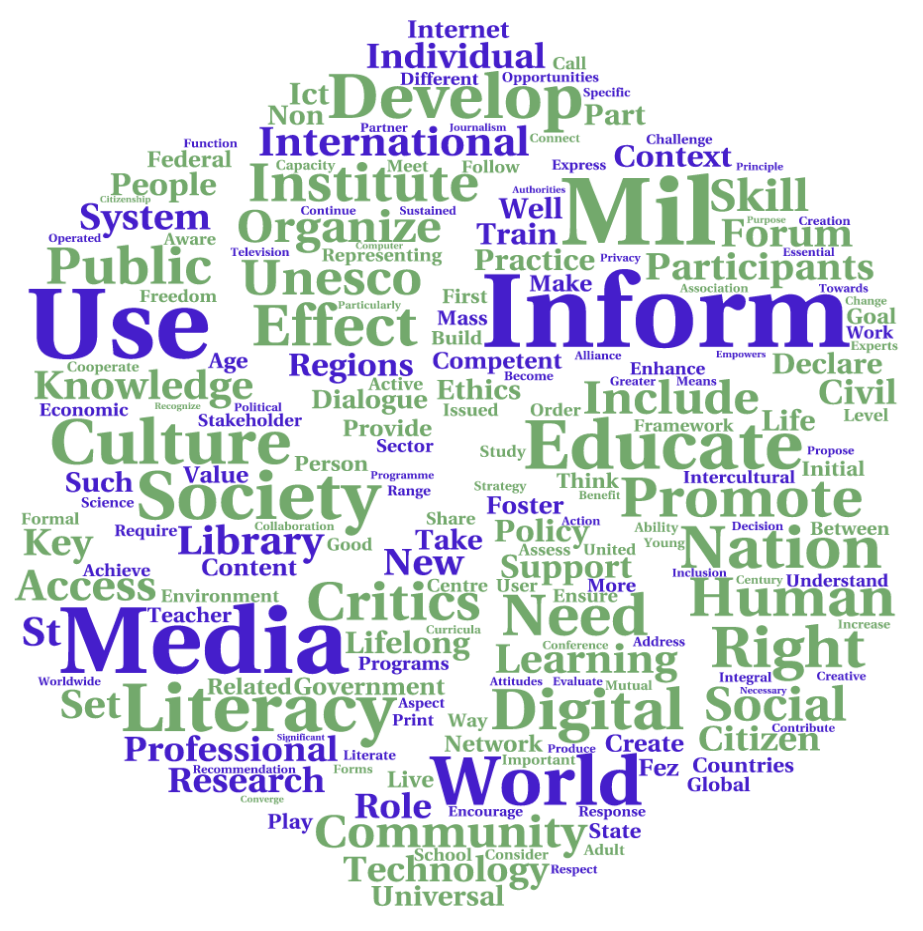

Fuente: Elaboración propia.

En las declaraciones más recientes (Fez, Moscú y París), los sitios de medios sociales y redes sociales adquieren relevancia, ya que sirven como referencias a las nuevas formas de interacción, así como, los nuevos derechos y modelos de participación para una ciudadanía global en la era digital. De forma general hacen un llamado a fomentar el rol de creadores críticos y a los actores clave y responsables políticos, a reflexionar más profundamente, cooperar y promover la AMI para todos.

La AMI deriva de iniciativas promovidas desde finales del siglo XX. Constituye una propuesta integradora que la UNESCO presenta en el nuevo siglo como derecho humano fundamental para enfrentar la convergencia de las Tecnologías de la Información y la Comunicación (TIC) y construir una cultura de paz sobre la base del diálogo intercultural, el respeto mutuo, la participación y entendimiento de todos.

Los fundamentos de la AMI tienen sus bases en la educación en medios de principios de la década del 80. Su campo adquiere un alcance mayor a partir de la integración de los principios de la Alfabetización 
Mediática y la Alfabetización Informacional, en la primera década del siglo XXI (DECLARACIÓN..., 2012).

Las primeras referencias aluden al empoderamiento de “...las personas en todos los ámbitos de la vida para buscar, evaluar, utilizar y crear la información de una forma eficaz para alcanzar sus metas personales, sociales, ocupacionales y educativas" como derecho básico en un mundo digital que promueve la inclusión social de todas las naciones (DECLARACIÓN..., 1982). Se enmarcan en el ámbito de la Alfabetización Mediática y desde entonces apunta a la relación con la Alfabetización Informacional.

Lee y So (2014), reflexionan sobre la reciprocidad existente entre los alfabetismos que constituyen pilares del concepto. Analizan las dos escuelas de pensamiento que emergen en la concepción de la AMI. Para algunos autores, la alfabetización informacional es un campo de estudio más amplio, en dónde la alfabetización mediática forma parte de esta, mientras que para otros, la alfabetización informacional es solamente una parte de la alfabetización mediática, la cual se ve como un campo más amplio.

Su concepción manifiesta la evolución hacia una consolidación e integración de varios alfabetismos como una única serie de competencias, (conocimientos, habilidades y actitudes) que permiten identificar las funciones de los medios de comunicación y de los dispositivos de información en la vida cotidiana. Apuntan hacia un aprendizaje que favorezca la comprensión, la interpretación y el pensamiento crítico a partir del uso de medios e información. Reconoce el papel de los proveedores de información como bibliotecas, archivos, museos e Internet, independientemente de las tecnologías utilizadas en la sociedad.

Según Wilson (2012), no se trata solo de la adquisición de habilidades técnicas, sino que comprende también el desarrollo de marcos y acercamientos de sentido crítico para evaluar los medios de comunicación y las fuentes de información, estudiando en particular, cómo se crean y cómo se transmiten los mensajes y cuál es la audiencia prevista. Incluye una concientización sobre el derecho al acceso a la información, así como, la importancia del uso ético de las tecnologías de la información para comunicarse con los otros. Incentiva el uso responsable de los medios y de la tecnología que desplaza a los usuarios desde la autonomía crítica a la solidaridad crítica.

La AMI reconoce la individualidad en los procesos de interpretación, negociación, aceptación o rechazo de mensajes e información, sustentados en la edad, la cultura, las experiencias de vida, los valores y creencias. Reafirma la libertad de expresión y de información, pues posibilita que las personas se eduquen según sus necesidades más primordiales. Su alcance trasciende a las áreas de ciudadanía activa, el debate democrático, la participación social y el empoderamiento (WILSON, 2012)

En los conceptos anteriormente tratados se respetan las particularidades de cada alfabetismo. Esto repercute en su concepción multidisciplinar que aúna perspectivas provenientes de los estudios sociales de la Tecnología, Ciencias de la Información y Documentación, la Educación, la Lingüística o el ámbito de la Comunicación y Estudios de Audiencias, principalmente. 


\section{Consideraciones finales}

La pluralidad de los alfabetismos que se manifiesta a principios del siglo XXI evoluciona hacia la síntesis y clasificación de la diversidad de términos bajo un denominador común. La Alfabetización Mediática y la Alfabetización Informacional se destacan por su auge en la literatura que aborda el tema, mientras, la Alfabetización Multimedia constituye un alfabetismo menos abordado. La noción de Alfabetización Mediática e Informacional (AMI) constituye la propuesta que integra los alfabetismos, reconociendo sus individualidades a partir del énfasis en las competencias (conocimientos, habilidades y actitudes) vinculadas a las funciones de los medios de comunicación y de los dispositivos de información en la vida cotidiana.

\section{Referencias}

AGUADED, J. I. El Parlamento Europeo apuesta por la alfabetización mediática. Comunicar, v. 32, n. 16, p. 7-8, 2009.

AGUADED, J. I. Apuesta de la ONU por una educación y alfabetización mediáticas. Comunicar, v. 38, n. 19, p. 7-8, 2012.

AGUADED, J. I. El Programa «Media» de la Comisión Europea, apoyo internacional a la educación en medios. Comunicar, v. 40, n. 20, p. 7-8, 2013.

ALBERICH, J. et al. Comunicación audiovisual digital: nuevos medios, nuevos usos, nuevas formas. Barcelona: Editorial EOC, 2005.

ALBERICH PASCUAL, J. et al. Comunicación audiovisual digital: nuevos medios, nuevos usos, nuevas formas. Barcelona: Editorial EOC, 2005.

ANDRETTA, S. Transliteracy: take a walk on the wild side. In: IFLA GENERAL CONFERENCE AND COUNCIL. Word Library and Information Congress, 75., Italia. Anais... Milán, Italia: IFLA, 2009. p. 1-14.

APARICI, R. Medios de comunicación y educación. Revista de Educación, n. 338, p. 85-99, 2005. Disponible en: <http://www.revistaeducacion.mec.es/re338/re338 07.pdf>. Consultado: 24 nov. 2013

APARICI, R.; GARCÍA, A. Lectura de imágenes en la era digital. Madrid: Ediciones de la Torre, 2008.

AREA, M.; PESSOA, T. De lo sólido a lo líquido: las nuevas alfabetizaciones ante los cambios culturales de la Web 2.0. Comunicar, v. 38, n. 19, p. 13$20,2012$.

BAUTISTA, A. Alfabetización tecnológica multimodal e intercultural. Revista de Educación, n. 343, p. 589-600, 2007.

BAWDEN, D. Revisión de los conceptos de Alfabetización Informacional y Alfabetización Digital. Anales de la Documentación, n. 5, p. 361-408, 
2002.

$<$ http://revistas.um.es/analesdoc/article/download/2261/2251>. Acesso em: 15 enero 2009.

BYRNE, A. La Afabetización informacional desde una perspectiva global: el desastre agudiza nuestras mentes. Anales de la Documentación, n. 8, p. 7-20, 2005.

CABERO, J.; GUERRA, S. La Alfabetización y formación en medios de comunicación en la formación inicial del profesorado. Educación XXI, v. 14, n. 1, p. 89-115, 2011.

CAMARGO, C. F. Multimedia Literacy in EFL Teacher Training. s.f. Disponível em: $<$ http://www.ohanalearning.org/wp/WP CFCMultimedia.pdf $>$. Acesso em: 14 abr. 2015.

CAZDEN, C. et al. A pedagogy of multiliteracies: Designing social futures. Harvard Educational Review, v. 66, n. 1, p. 60-96, 1996.

CORDES, S. Broad horizons: the role of multimodal literacy in 21st century library instruction. In: IFLA GENERAL CONFERENCE AND COUNCIL, 75., Milan, Italia, 2009. Word Library and Information Congress. Italia: [s.n.], 2009.

DALEY, E. Expanding the concept of literacy. Educase review, v. 38, n. 2, p. 32-40, 2003.2 Disponível em: $<$ http://iml.usc.edu/downloads/news articles/erm0322.pdf $>$. Acesso em: 14 abr. 2015.

DECLARACIÓN de Alejandría: Faros de la Sociedad de la Información: Proclamación de Alejandría acerca de la Alfabetización Informacional y el aprendizaje de por vida. Alejandría, 2005. Disponible en: <http://www.ifla.org/node/7275>. Consultado: 8 oct. 2013.

DECLARACIÓN de Grünwald sobre la educación relativa a los medios de comunicación. Grünwald, $1982 . \quad$ Disponible en: <http://www.unesco.org/education/nfsunesco/pdf/MEDIA_S.PDF>.

Consultado: 8 oct. 2013.

DECLARACIÓN de Lyon sobre el acceso a la información y el desarrollo. Lyon, 2014.2 Disponible en: $<$ http://blogs.ifla.org/lac/2014/08/declaracion-de-lyon-version-enespanol/>. Consultado: 3 feb. 2015.

DECLARACIÓN de Moscú sobre Alfabetización Mediática e Informacional. Moscú, $2012 . \quad$ Disponible en: $<$ http://www.unesco.org/new/fileadmin/.../moscow_declaration_mil_es.p df>. Consultado: 8 oct. 2013.

DONDIS, D. A. La sintaxis de la imagen: introducción al alfabeto visual. España: Ed. Gustavo Gili, 2003. 
DUDZIAK, E. A. Information literacy: princípios, filosofia e prática. Ciencias da Informacao, v. 32, n. 1, p. 23-35, 2003. Disponível em: $<$ http://www.ibict.br/cionline/320103/32010303.pdf >. Acesso em: 30 abr. 2009.

ERSTAD, O. Electracy as empowerment: student activities in learning environments using technology. Young, v. 11, n. 11, p. 11-28, 2003. Disponível em: <http://you.sagepub.com/cgi/content/abstract/11/1/11 > Acesso em: 30 abril 2009.

FERREIRO, E. Librarians and basic education teachers in the context of 'Digital Literacy'. IFLA Journal, v. 31, n. 1, p. 35-44, 2005. Disponível em: <http://ifl.sagepub.com/cgi/reprint/31/1/35 >. Acesso em: 5 feb. 2014.

FEZ Declaration on Media and Information Literacy. Fez 2011. Disponible en:

<http://www.unesco.org/new/fileadmin/MULTIMEDIA/HQ/CI/CI/pdf/news/ Fez\%20Declaration.pdf>. Consultado: 8 oct. 2013.

GENZUK, M. Visions of possibilities: multimedia literacy in teacher education. UrbanEd: The Journal of the Rossier School of Education, $\mathrm{n}$. Spring/Summer, p. 18-21, 2005. Disponível em: < $\underline{\text { http://www- }}$ rcf.usc.edu/ genzuk/UrbanEdMagLayoutM GenzukFullPage.pdf >. Acesso em: 30 abr. 2009.

GUTIÉRREZ, A. Alfabetización Multimedia. 2000. Disponível em: $<$ http://www.doe.uva.es/alfonso/web/IndAlfMult.htm>. Acesso em: 3 de diciembre.

GUTIÉRREZ, A.; TYNER, K. Alfabetización mediática en contextos múltiples. Comunicar, v. 19, n. 38, p. 10-12, $2012 \mathrm{a}$.

GUTIÉRREZ, A.; TYNER, K. Educación para los medios, alfabetización mediática y competencia digital. Comunicar, v. 19, n. 38, p. 31-39, 2012b.

GUTIERREZ, A. Multimedia authoring as a fundamental principle of literacy and teacher training in the information age. In: DUNCAN, B.; TYNER, K. (Ed.). Visions, revisions: moving forward with Media Education. Madison: National Telemedia Council, 2003.

GUTIÉRREZ, A. La Educación multimedia: una propuesta desmitificadora. Diálogos de la comunicación, p. 52-65, 1998.

GUTIÉRREZ MARTÍN, A. Alfabetización multimedia. 2000a. Disponível em: <http://www.doe.uva.es/alfonso/web/IndAlfMult.htm >. Acesso em: 3 de diciembre.

GUTIÉRREZ MARTÍN, A. Educación Multimedia: nuevos textos, nuevos contextos. In: AA., V. (Ed.). Pedagogía viva. Madrid: Ed. C.P.R. de Arganda del Rey, 2000b. 
GUTIÉRREZ MARTÍN, A. Influencia del discurso tecnológico en la integración curricular de las nuevas tecnologías multimedia: Universidad Nacional de Educación a Distancia, Facultad de Ciencias de la Educación, 2000c.

GUTIÉRREZ, A. Alfabetización digital: algo más que ratones y teclas. Barcelona: Gedisa Editoria, 2003.

GUTIÉRREZ MARTÍN, A.; HOTTMANN, A. Democracy, Multimedia Literacy and classroom practice: a european experience. Berlin: Mondial Verlag, 2002.

HARTLEY, J. There are other ways of being in the truth: the uses of multimedia literacy. International Journal of Cultural Studies, v. 10, n. 1 , p. 135-144, 2007. Disponível em: $<$ http://ics.sagepub.com/cgi/reprint/10/1/135>. Acesso em: 11 nov. 2009.

HARTLEY, J. et al. The uses of multimedia: three Digital Literacy case studies. Media International Australia, n. 128, 2008.

HATTWIG, D. et al. Visual Literacy Standards in Higher Education: new opportunities for libraries and student learning. Libraries and the Academy, v. 13, n. 1, p. 61-89, 2013.

HOBBS, R. Multiple visions of Multimedia Literacy: emerging areas of synthesis. Handbook of literacy and technology, v. 2, p. 15-28, 2006.

HOBBS, R.; JENSEN, A. The past, present, and future of Media Literacy Education. Journal of Media Literacy Education, n. 1, p. 1-11, 2009.

HOFSTETTER, F. T. Multimedia Literacy. New York: Irwin/McGraw-Hill, 2001.

IFLA. Recomendaciones IFLA sobre Alfabetización Informacional y Mediática. 2011.

Disponible: <http://bivir.uacj.mx/dhi/7e/docstaller1/2011_IFLA_Unesco_guidelines_E spa_DHI_JLau_enero.pdf>. Consultado: 29 mar. 2012.

KELLNER, D.; SHARE, J. Toward Critical Media Literacy: core concepts, debates, organizations, and policy, Discourse: studies in the cultural politics of education. Discourse: Studies in the Cultural Politics of Education, v. 26, n. 3, p. 369-386, 2005.

KULTHAU, C. Implementing a Process Approach to Information Skills: A Study Identifying Indicators of Success in Library Media Programs. School Library Media Quarterly, v. 22, n. 1, 1993. Disponível em: $<$ http://www.ala.org/aasl/SLMR/slmr resources/select kuhlthau1.html >. Acesso em: 23 noviembre 2006.

LAU, J. Information skills: conceptual convergence between Information and Communication Sciences. In: UNESCO (Ed.). Media and Information Literacy for knowledge societies. Moscú: Interregional Library Cooperation Centre, 2013. p. 63-77. 
LEE, A. Y. L.; SO, C. Y. K. Alfabetización Mediática y Alfabetización Informacional: similitudes y diferencias. Comunicar, v. 21, n. 42, p. 137146, 2014. Disponível em: < www.revistacomunicar.com >. Acesso em: 5 nov. 2013.

LEMKE, J. Multimedia Literacy demands of the scientific curriculum. Linguistics and Education, v. 10, n. 3, p. 247-271, 1998.

LEMKE, J. Towards critical Multimedia Literacy: Technology, research, and politics. In: MCKENNA, M. et al. (Ed.). Handbook of literacy \& technology. Erlbaum: LEA Publishing, 2005. Disponible: <http://wwwpersonal.umich.edu/ jaylemke/papers/reinking2.htm $>$. Consultado: 5 nov. 2013

LONSDALE, M.; MCCURRY, D. Literacy in the new millennium. Australia: NCVER, 2004.

MACKEY, T. P.; HO, J. Implementing a convergent model for information literacy: combining research and web literacy. Journal of Information Science, v. 31, n. 6, p. 541-555, 2005.

MACKEY, T. P.; JACOBSON, T. E. Reframing Information Literacy as a Metaliteracy College \& Research Libraries, v. 76, n. 1, p. 62-78, 2011.

MCPHERSON, K. Multiplying literacies in school libraries. Teacher Librarian, v. 32, n. 1, p. 60-62, 2004.

MESSARIS, P. Visual aspects of Media Literacy. Journal of Communication, v. 48, n. 1, p. 70-80, 1998.

NATHARIUS, D. The More We Know, the More We See: the Role of Visuality in Media Literacy. American Behavioral Scientist, v. 48, n. 2, p. 238-247, 2004.

NEGROPONTE, N. El mundo digital. Barcelona: Ediciones B, 1995.

PARIS Declaration on Media and Information Literacy in the Digital Era. Paris 2014. Disponible en: <http://www.unesco.org/new/fileadmin/MULTIMEDIA/HQ/CI/CI/pdf/news/ paris_mil_declaration.pdf>. Consultado: 12 enero 2015.

PASADAS, C. Multialfabetismo y alfabetización informacional crítica: marco de referencia para la función educativa de la biblioteca. In: GÓMEZ, J. A. et al. (Ed.). Brecha digital y nuevas alfabetizaciones: el papel de las bibliotecas. Madrid: Biblioteca de la Universidad Complutense de Madrid, 2008. p. 59-85.

PAUL, P. V. New literacies, multiple literacies, unlimited literacies: what now, what next, where to?: a response to Blue Listerine, Parochialism and ASL Literacy. Journal of Deaf Studies and Deaf Education, v. 11, n. 3, p. 382-387, 2006.

PEDROSO, T. De la retórica a la seducción. Alfabetización en Publicidad. Ágora digital, n. 5, p. 1-9, 2003. Disponível em: < http://hdl.handle.net/10272/3510 >. Acesso em: 4 febrero 2011. 
PINTO, M. et al. Alfabetización múltiple desde la biblioteca pública: experiencias y propuestas. Buenos Aires: Alfagrama, 2009.

PONJUAN, G. De la alfabetización a la cultura informacional: rol del profesional de la información. CONGRESO INTERNACIONAL DE INFORMACIÓN INFO 2002, 7., 2002, Havana. Anales... Cuba: INFO, 2002. IDICT.

THE PRAGUE Declaration: Towards an information literate society. Praga 2003.

Disponible: <http://www.unesco.org/new/fileadmin/MULTIMEDIA/HQ/CI/CI/pdf/Pragu eDeclaration.pdf>. Consultado: 12 junio 2014.

SANTOS, E. M. D.; ANDRADE, E.; VIDAL, N. Cidadania e trabalho na sociedade da informação: uma abordagem baseada na competência. Perspectivas em Ciência da Informação, v. 13, n. 3, p. 208-222, 2008.

THOMAS, S. et al. Transvliteracy: Crossing divides. First Monday, v. 12, n. 12, 2007.2 Disponível em: <http://www.uic.edu/htbin/cgiwrap/bin/ojs/index.php/fm/article/view/206 $\underline{0 / 1908}$ >. Acesso em: 5 nov. 2013.

TYNER, K. Audiencias, intertextualidad y nueva alfabetización en medios. Comunicar, v. 15, n. 30, p. 79-85, 2008.

UNESCO. La alfabetización, un factor vital: informe de seguimiento de la EPT en el mundo. Paris: Ediciones UNESCO, 2006.

URIBE, A. La alfabetización informacional en las bibliotecas universitarias de Brasil: visualización de los niveles de incorporación desde la información publicada en sus sitios Web. Perspectivas em Ciência da Informação, v. 17, n. 1, p. 134-152, 2012.

WILSON, C. Alfabetización mediática e informacional: proyecciones didácticas. Comunicar, v. 39, n. 20, p. 16-24, 2012.

ZAIN, A. M.; YEW LIE, K. Weblogging as a Multimedia Literacy event: analyzing the discourse space of a student blogger's speech community. Malaysian Journal of ELT Research, v. 5, p. 1-26, 2009. Disponível em: $<$ http://www.melta.org.my/modules/tinycontent/Dos/Azni MOhd Zain 2 009.pdf >. Acesso em: 5 nov. 2013. 